\title{
PREVALÊNCIA E ASPECTOS EPIDEMIOLÓGICOS DA LEPTOSPIROSE BOVINA EM REBANHO LEITEIRO NA MICRORREGIÃO DE GOIÂNIA - GO
}

\author{
PREVALENCE AND EPIDEMIOLOGY ASPECTS OF BOVINE LEPTOSPIROSIS \\ IN DAIRY HERD FROM GOIÂNIA MICROREGION, \\ GOIÁS STATE, BRAZIL
}

\author{
Raquel Soares Juliano ${ }^{1}$ Nilo Sérgio Troncoso Chaves ${ }^{2}$ Cléverson Acyprestes dos Santos ${ }^{1}$ \\ Ludmila Souza Ramos ${ }^{1}$ Hélvio Queiroz dos Santos ${ }^{1}$ Luciana Rodrigues Meireles ${ }^{3}$ \\ Suzana Gottschalk ${ }^{3}$ Ruy Alberto Caetano Corrêa Filho ${ }^{4}$
}

RESUMO

Foram colhidas 426 amostras de hemossoro bovino, provenientes de vacas em lactação na microrregião de Goiânia$G O$, e testadas pelo método de soroaglutinação microscópica (SAM) para detecção de aglutininas antileptospiras, para efetuar um estudo de prevalência. Paralelamente, foi realizado um questionário epidemiológico, contendo informações que pudessem fornecer dados sobre a epidemiologia da leptospirose nessa região. Os resultados obtidos revelaram uma prevalência de $81,90 \%$ de animais reagentes. Os principais sorovares envolvidos foram: wolffi $(36,10 \%)$, icterohaemorrhagiae (20,50\%), hardjo $(5,20 \%)$ e tarassovi $(4,90 \%)$. A presença de ratos e suínos, a utilização de inseminação artificial e o tipo de fonte de água não interferiram no número de animais reagentes no teste de SAM. A leptospirose tem um comportamento enzoótico nessa região.

Palavras-chave: leptospirose bovina, prevalência, epidemiologia, gado de leite.

\section{SUMMARY}

With the aim of search the prevalence of antileptospiral antibodies, 426 sera samples were collected from dairy cattle in the microregion of Goiania-GO, Brazil. Some questions were made about epidemiological and sanitary conditions characteristics of the farms to help us to understand the behavior of leptospirosis in this region. The prevalence was $81.90 \%$ and the serovars more involved were: wolffi $(36.10 \%)$, icterohaemorrhagiae (20.50\%), hardjo (5.20\%) and tarassovi (4.90\%). There were pigs and rats in the farms, but they did'nt interfer on positive animals number. This afirmative is also correct to artificial insemination and water supplying. The bovine leptospirosis had enzootic characteristics in this region.

Key words: bovine leptospirosis, prevalence, epidemilogy, dairy cattle.

\section{INTRODUÇÃO}

A leptospirose em humanos foi descrita, em 1886 e chamada Doença de Weil; o agente etiológico, entretanto, só foi isolado em 1915, por Inada e Ido, no Japão. Noguchi, em 1917, estabeleceu finalmente o gênero Leptospira, isolando o microorganismo de um rato, pela primeira vez (STEELE, 1957).

A leptospirose é uma zoonose e afeta várias espécies de animais domésticos e silvestres, que podem funcionar como portadores (sadios ou doentes) ou reservatórios. A transmissão se dá por contato direto com a urina, sangue ou tecidos de animais infectados, ou de modo indireto pela água e/ou alimentos contaminados. Animais em lactação podem eliminar leptospiras no leite na fase aguda da doença. As vias de penetração no homem e animais são a pele lesada, mucosa orais, nasais, oculares e genitais

\footnotetext{
${ }^{1}$ Médica Veterinária, mestrando da Escola de Veterinária (EV), Universidade Federal de Goiás (UFG), Rua Marquês de Leão, 562 casa 09, 79034-520, Campo Grande, MS. Autor para correspondência

${ }^{2}$ Professor Doutor, Departamento de Medicina Veterinária, EV, UFG, Goiânia, GO.

${ }^{3}$ Médica Veterinária, residente do Depto. de Hig. Vet. e Saúde Pub. FMVZ-UNESP-Botucatu, Rubião Júnior-SP.

${ }^{4}$ Professor, Doutor, Depto. de Produção Animal da E.V.-UFMS, Av. Filinto Müler s/n, Campo Grande-MS
} 
(nos animais). O período de incubação é em média de dois a cinco dias e as leptospiras são eliminadas na urina de animais infectados, logo após a bacteremia. Isso ocorre, teoricamente, da segunda a quinta semana da doença; mas animais convalescentes podem eliminá-las durante meses (MANUAL DE CONTROLE DA LEPTOSPIROSE, 1989).

A importância da leptospirose é relevante, pois, além de ser uma zoonose, causa consideráveis perdas econômicas no rebanho bovino, devido a abortos, retenção de placenta, nascimentos prematuros, morte, infertilidade, decréscimo na produção de leite e problemas com mastite (ELLIS, 1984; LANGENEGGER, 1990).

Os objetivos deste trabalho foram determinar a prevalência da leptospirose em rebanhos leiteiros na microrregião de Goiânia-GO, identificar quais os sorovares de leptospiras, dentre aqueles mais frequientemente associados à infecção em bovinos, estão presentes em fêmeas lactantes e obter informações sobre alguns fatores que interferem na epidemiologia da leptospirose nessa região.

\section{MATERIAL E MÉTODOS}

A microrregião de Goiânia está situada entre as latitudes $16^{\circ} 16^{\prime}$ e $17^{\circ} 13^{\prime} \mathrm{S}$ e os meridianos $48^{\circ} 38^{\prime} \mathrm{e} 49^{\circ} 45^{\prime} \mathrm{W} \mathrm{Gr}$, sendo formada por 17 municípios. O clima é classificado como tropical com duas estações distintas; o verão chuvoso, com precipitações máximas de $300 \mathrm{~mm}$ e o inverno seco, com precipitações mínimas de $11 \mathrm{~mm}$. O índice pluviométrico anual é de $1533,4 \mathrm{~mm}$ e a temperatura oscila entre 18 e $26^{\circ} \mathrm{C}$ (1995). Essa microrregião possui cerca de 119.743 vacas em ordenha e uma produtividade média de 86.197.000 litros de leite por ano. A área total da microrregião é de $6.847,9 \mathrm{~km}^{2}$, caracterizada por possuir propriedades leiteiras de pequeno e médio porte (ANUÁRIO ESTATÍSTICO DO ESTADO DE GOIÁS, 1996).

O número de amostras foi calculado , utilizando-se a fórmula proposta pelo CENTRO PANAMERICANO DE ZOONOSES (1973), com uma prevalência esperada de $20 \%$ (JARDIM et al., 1978), grau de confiança igual a $95 \%$ e o erro não ultrapassando $20 \%$ da prevalência. Estabeleceu-se um número de até 25 animais por propriedade, ou $20 \%$ do número de vacas em lactação. No total, foram coletadas 426 amostras de 20 propriedades. Os municípios foram sorteados aleatoriamente com reposição, considerando-se que cada vez que ele fosse sorteado, seria o equivalente a uma propriedade, onde a colheita de amostra seria realizada. O período de coleta foi de janeiro a março de 1998.
As amostras de sangue foram obtidas por punção da veia caudal dos animais, acondicionadas em tubos de vidro de $20 \mathrm{~m} \ell$ e identificadas. Logo após a colheita, o material foi levado ao laboratório de sorologia da Escola de Veterinária da UFG e centrifugado em 2500rpm durante cinco minutos. O soros foram então colocados em tubos plásticos $(1,5 \mathrm{~m} \ell)$ e congelados a $-20^{\circ} \mathrm{C}$ até o momento da realização do teste de soroaglutinação microscópica (SAM).

Foi estruturado e aplicado um questionário epidemiológico para cada propriedade rural onde as colheitas foram realizadas, na tentativa de recolher informações para o estudo epidemiológico da leptospirose bovina na região.

Os testes de soroaglutinação microscópica (SAM) foram realizados no laboratório de diagnóstico de leptospirose do Departamento de Higiene Veterinária e Saúde Pública da Faculdade de Medicina Veterinária e Zootecnia - UNESP-Botucatu, seguindo as normas técnicas propostas pelo Ministério da Saúde (MANUAL DE CONTROLE DA LEPTOSPIROSE, 1989), onde, primeiramente, foi feita a triagem das amostras para cada sorovar e considerados reagentes as amostras que aglutinaram $50 \%$ ou mais das leptospiras vivas contidas na solução antigênica. Posteriormente, essas amostras reagentes foram testadas novamente para definir o título de anticorpos para cada sorovar; que neste caso variou de 1:100 a 1:3200. Da mesma forma, foram considerados títulos de amostras que aglutinaram $50 \%$ ou mais das leptospiras vivas contidas na solução antigênica. A leitura das reações de aglutinação foi feita em microscópio de campo escuro. Os sorovares utilizados foram: bratislava, castellonis, canicola, djasiman, grippotyphosa, copenhageni, icterohaemorrhagiae, pomona, pyrogenes, hardjo, wolffi e tarassovi.

Os resultados obtidos foram descritos em porcentagem, calculando-se a prevalência da microrregião para os 12 sorovares de Leptospira interrogans, testados por SAM. A correlação entre a prevalência e algumas variáveis (presença de abortos e retenção de envoltórios fetais, presença de ratos, tipo de fonte de água, utilização de inseminação artificial e presença de suínos) foi feita pelo teste de quiquadrado $\left(\chi^{2}\right)$.

\section{RESULTADOS E DISCUSSÃO}

A porcentagem de animais reagentes $(81,90 \%)$, encontrada no rebanho amostrado na microrregião de Goiânia, está acima dos resultados descritos por JARDIM et al. (1978), que encontrou 20,57\% de positividade em 418 amostras obtidas em 
diversos municípios de Goiás. Houve então um acentuado aumento da prevalência nos últimos 20 anos, porém devem ser considerados alguns fatores: O rebanho amostrado por JARDIM et al. (1978) não era procedente exclusivamente da microrregião de Goiânia-GO, não havendo informações adicionais sobre os animais ou as propriedades amostradas; houve consideráveis modificações no rebanho leiteiro do Estado de Goiás nos últimos 20 anos no que diz respeito ao número de animais, técnicas de manejo, introdução de material genético e trânsito de animais de outras localidades. Dessa forma, qualquer tipo de avaliação seria inconsistente.

Em outros Estados, os resultados mais próximos foram obtidos por MADRUGA et al. (1980), que descreveram $74,30 \%$ de positividade para amostras obtidas no Estado do Mato Grosso do Sul. Esses mesmos autores encontraram amplitude térmica entre 18,30 e $28,90^{\circ} \mathrm{C}$ e precipitações pluviométricas entre 1400 e $1600 \mathrm{~mm}$, destacando estas condições como favoráveis à disseminação da leptospirose no cerrado. A microrregião de Goiânia possui condições climáticas muito semelhantes, com precipitação pluviométrica média de $1533,40 \mathrm{~mm}$ anuais e amplitude térmica entre 18 e $26^{\circ} \mathrm{C}$, o que poderia explicar tal semelhança na prevalência da leptospirose nesses dois Estados. VIEGAS et al. (1986) citaram 68,90\% em dois municípios do Estado da Bahia. A prevalência registrada por LOPEZ et al. (1990), no Chile, foi de 53,76\%; menor do que o resultado encontrado neste estudo sorológico da microrregião de Goiânia. Entretanto, os autores utilizaram o título de 1:200 como "cut-off", o que pode ter subestimado os resultados.

A porcentagem de animais reagentes ao teste de SAM para cada sorovar está descrita na tabela 1. A predominância de reações aglutinantes para o sorovar wolffi $(29,60 \%)$ também foi descrita anteriormente, no Estado de Goiás, por JARDIM $\boldsymbol{e t}$ al. (1978), que encontraram 50\% de prevalência, e apareceu nos resultados de vários estudos de prevalência realizados no Brasil na década de 70: CORDEIRO et al. (1975) no Rio de Janeiro; DORIA \& SANTANA (1976) e CALDAS et al. (1977) na Bahia e OLIVEIRA (1977) no Rio Grande do Sul.

Segundo LILENBAUM (1996), essa estatística modificou-se a partir da década de 80 , com a inclusão do sorovar hardjo na bateria de testes do sorovar SAM. Foi desde então que este sorovar vem sendo estudado com maior profundidade, sendo considerado como prevalente na espécie bovina. Mesmo assim, alguns rebanhos mantiveram a prevalência de wolffi como sorovar mais freqüente: DORIA et al. (1982), com 48,47\% no Estado da Bahia e em dois rebanhos no estado de Goiás (CAMPOS JR. \& FRENEAU, 1996).
Tabela 1 - Número e porcentagem de animais reagentes aos sorovares de $\boldsymbol{L}$. interrogans, no teste de SAM, em 426 soros bovinos da microrregião de Goiânia, GO, 1998.

\begin{tabular}{lc}
\hline \multicolumn{1}{c}{ SOROVAR } & $\begin{array}{c}\text { NÚMERO DE ANIMAIS / } \\
\text { \% REAGENTES }\end{array}$ \\
& \\
\hline & \\
wolffi & $(\mathrm{n}=126) 36,10 \%$ \\
icterohaemorrhagiae & $(\mathrm{n}=72) 20,50 \%$ \\
hardjo & $(\mathrm{n}=18) 5,20 \%$ \\
tarassovi & $(\mathrm{n}=17) 4,90 \%$ \\
bratislava & $(\mathrm{n}=11) 3,10 \%$ \\
pomona & $(\mathrm{n}=8) 2.30 \%$ \\
djasiman & $(\mathrm{n}=8) 2,30 \%$ \\
pyrogenes & $(\mathrm{n}=7) 2,00 \%$ \\
copenhageni & $(\mathrm{n}=3) 0,90 \%$ \\
castelonis & $(\mathrm{n}=3) 0,90 \%$ \\
canicola & $(\mathrm{n}=1) 0,30 \%$ \\
grippotyphosa & $(\mathrm{n}=1) 0,30 \%$ \\
Co-aglutinações & $(\mathrm{n}=74) 21,20 \%$ \\
& \\
\hline
\end{tabular}

TOTAL

$(n=349) 100 \%$

No Brasil, o sorovar hardjo foi predominante em rebanhos dos Estados do Sul, Sudeste e Centro-Oeste (VASCONCELLOS, 1997). O mesmo ocorreu em estudos realizados na Nigéria, com 35,60\% (EZEH et al., 1990), nos Estados Unidos e Porto Rico, com 83\% (MILLER, WILSON, BERAN,1991), em Portugal (PEREIRA \& ROCHA, 1991) e na Austrália (ABDOLLAHPOUR, et al. 1996). Essa afirmativa não pode ser adotada para o rebanho da microrregião de Goiânia, que obteve prevalência de apenas 5,20\% para hardjo. Mesmo considerando a possibilidade de reações cruzadas entre hardjo e wolffi, a prevalência do segundo foi aproximadamente sete vezes maior e ficou clara sua predominância no rebanho estudado.

A presença do sorovar icterohaemorragiae como segundo sorovar mais prevalente $(20,60 \%)$ difere do resultado encontrado por JARDIM et al. (1978), que foi 5,80\%, no Estado de Goiás. Encontrou-se um valor aproximado, 45,69\%, no Valle do Atlixco, no México (LUNA, et al. 1993). Não há qualquer citação sobre a importância desse sorovar para os rebanhos estudados por esses autores, porém icterohaemorragiae já havia sido isolado anteriormente de feto bovino abortado e teve papel relevante como agente etiológico da "Doença de Weil" em humanos (SANTA ROSA et al., 1961).

A prevalência do sorovar tarassovi e bratislava na microrregião de Goiânia foi de 4,90\% e $3,10 \%$, respectivamente. A presença de tarassovi já havia sido descrita no Estado do Rio de Janeiro, 
apresentando 2,62\% (CORDEIRO et al., 1975), na Bahia, com prevalência variando entre 9,93 e 22,50\% (CALDAS et al., 1977; DORIA et al.,1982; VIEGAS et al., 1986) e Rio Grande do Sul, 0,75\% (ABUCHAIM \& DUTRA, 1985).

A ocorrência de aglutinações para cada diluição foi, em sua maioria, em baixas diluições. Somente oito reações de aglutinação ocorreram com título 1:3200.

A dinâmica dos sorovares para cada título aglutinante demonstrou que icterohaemorragiae, wolffi, hardjo e bratislava alternaram sua prevalência, entre os quatro sorovares mais freqüentes, nas diluições 1:100 e 1:200, enquanto que os outros sorovares ocorreram em freqüências muito menores. À medida que o título de anticorpos aglutinantes aumentou, ficou clara a prevalência de wolffi.

A ocorrência de aglutinações, em sua grande maioria, nas diluições 1:100 e 1:200, indicou que a leptospirose é uma enfermidade enzoótica na microrregião de Goiânia, considerando afirmação semelhante feita por ORDUÑA et al., (1996) em estudo de prevalência realizado no México. MOREIRA et al. (1979) encontraram baixos títulos para determinados sorovares que eram sabidamente muito patogênicos (pomona, hardjo, icterohaemorragiae, canicola e grippotyphosa), semelhantes aos resultados obtidos neste experimento e sugeriu que subinfecções poderiam ter tido um efeito imunizante no rebanho. Essa hipótese também pode ser considerada neste estudo.

Dos 426 animais amostrados, 354 eram provenientes de propriedades com presença de ratos e 72 vieram de propriedades onde não existiam ratos. O número de animais reagentes que tiveram contato com ratos e não tiveram contato com ratos foi, respectivamente, 290 e 59. Animais reagentes para o sorovar icterohaemorragiae somam um total de 72 , sendo que 61 deles vieram de propriedades infestadas por ratos.

A análise de $\chi 2$ demonstrou que não houve correlação estatística entre a presença de ratos e a ocorrência de animais positivos para SAM nas propriedades da microrregião de Goiânia, mesmo quando se analisou separadamente essa relação com animais reagentes ao sorovar icterohaemorragiae. Deve, entretanto, ser levado em consideração que os roedores, domésticos e silvestres, são o maior reservatório desse sorovar. Sendo assim, o controle sistemático desses animais deve ser adotado como fundamental para a manutenção da sanidade do rebanho bovino e das pessoas que moram nessa região. Vários estudos confirmam a importância dos roedores como reservatório de icterohaemorragiae, dentre outros sorovares de leptospiras (CORDEIRO et al., 1981, KURIBARA et al., 1996) e o controle da sua população é uma prática recomendada (MANUAL DE CONTROLE DA LEPTOSPIROSE, 1989).

A prática da Inseminação Artificial (IA) foi realizada em 215 animais, dos quais 182 foram considerados reagentes no teste de SAM. Em contrapartida, 211 animais não foram submetidos a IA e destes, 167 foram reagentes. Dos 18 animais sororreativos, exclusivamente, para o sorovar hardjo, 13 não haviam sofrido IA e cinco eram provenientes de propriedades que praticavam IA no seu manejo reprodutivo.

Não houve diferença significativa entre a prevalência de animais sororreagentes no teste de SAM ou reagentes ao sorovar hardjo e a prática da IA, demonstrando que a IA não está correlacionada com estas variáveis nos animais amostrados na microrregião de Goiânia. Essas afirmações são contrárias às citações feitas por BROD \& FEHLBERG (1992) e BROD et al. (1995) sobre a epidemiologia da leptospirose bovina.

Em relação ao tipo de fonte de água oferecida aos animais, verificou-se que 282 animais vieram de propriedades que utilizavam bebedouros de superfície (água estagnada) com positividade em 236 animais, e 144 eram provenientes de propriedades cujo fornecimento de água aos animais era feito através de córregos e riachos (água corrente), apresentando positividade em 113 destes.

Através da análise de $\chi^{2}$ foi possível constatar que o tipo de fonte de água não deve ter afetado significativamente a ocorrência de animais reagentes nas propriedades da microrregião de Goiânia, discordando das afirmações feitas por GUIMARÃES (1982) e BROD \& FEHLBERG (1992), que citam a fonte de água como fator de risco importante na disseminação da leptospirose. Entretanto, em estudo posterior, realizado em rebanhos bovinos do Rio Grande do Sul, BROD et al. (1995) puderam afirmar que a fonte de àgua não estava relacionada com a presença da leptospirose nesses rebanhos.

Os abortos ocorreram em nove animais que apresentaram resultados positivos no teste de SAM e em dois animais não reagentes. A retenção de envoltórios fetais esteve presente em 27 dos 349 animais sororreagentes e em seis animais de um total de 77 soronegativos. A impossibilidade de avaliar a correlação de abortos e a não significância da retenção de envoltórios fetais com a prevalência de animais reagentes na microrregião de Goiânia dificulta uma análise conclusiva da importância dessas variáveis na epidemiologia da leptospirose das vacas lactantes da microrregião de Goiânia. Porém, diante do fato de ter sido encontrada uma prevalência elevada nesses animais e uma baixa freqüência na ocor- 
rência destes distúrbios reprodutivos (nove abortos e 27 retenções), sugeriu-se que a ocorrência de um grande número de animais sororreativos para leptospirose não está relacionada à ocorrência de abortos nesses animais amostrados. Esse fato pode ser explicado, pois a infecção por leptospiras nem sempre evolui para quadros clínicos reprodutivos, e depende da fase de gestação e da idade do animal infectado (LANGENEGGER, 1990).

Das propriedades coletadas, $67 \%$ apresentavam algum tipo de pecuária mista, mesmo que em pequena quantidade, criando principalmente suínos, aves e ovinos para consumo próprio. Dos 237 animais que tiveram contato com suínos, 18 foram reagentes para tarassovi e 11 para bratislava. Sendo assim, após análise de $\chi 2$, ficou estabelecido que não houve correlação entre a presença de suínos nas propriedades e o número de animais positivos para esses sorovares.

A prevalência do sorovar tarassovi foi associada a criações promíscuas entre bovinos e suínos no Rio de Janeiro e no Chile (CORDEIRO $\boldsymbol{e t}$ al., 1975; LOPEZ \& POQUET,1990). CISNEROS et al. (1996) citaram a ampla disseminação do sorovar bratislava também encontrado com freqüência em suínos da Europa e América Latina. Essa afirmação não foi comprovada neste estudo, apesar de $67 \%$ das propriedades apresentarem suínos, criados livremente em sua maioria.

\section{CONCLUSÕES}

As fêmeas bovinas lactantes, do rebanho leiteiro da microrregião de Goiânia, apresentaram prevalência alta de animais positivos ao teste de soroaglutinação Microscópica para leptospirose bovina. Os sorovares mais prevalentes foram wolffi, icterohaemorragiae, hardjo e tarassovi, sendo que a maioria das aglutinações ocorreram em diluições 1:100 e 1:200, sugerindo comportamento enzoótico da leptospirose bovina nessa microrregião. A presença de ratos e suínos, utilização de Inseminação Artificial e o tipo de fonte de água não interferiram no número de animais reagentes no teste de soroaglutinação Microscópica, nas propriedades amostradas na microrregião de Goiânia-GO, no presente estudo.

\section{REFERÊNCIAS BIBLIOGRÁFICAS}

ABDOLLAHPOUR, G., ENGLISH, A.W., TASLER, J. Isolation of Leptospira interrogans serovar grippotyphosa from a heifer in New South Wales. Aust Vet J, v.73, n.3, p.109-110, 1996.

ABUCHAIM, D.M., DUTRA, N.L.F. Prevalência da leptospiro- se em bovinos da bacia leiteira de Porto Alegre- RS. Arq Fac Vet UFRGS, v.13, p.55-60, 1985.

ANUÁRIO ESTATÍSTICO DO ESTADO DE GOIÁS. Secretaria do Planejamento e Desenvolvimento Regional - Superintendência de Estatística, Pesquisa e Informação. GoiâniaGO, 1996. 621p.

BROD, C.S., FEHLBERG, M.F. Epidemiologia da leptospirose em bovinos. Ciência Rural, v.22, n.2, p.239-245, 1992.

BROD, C.S., MARTINS, L.F.S., NUSSBAUM, A.A., $\boldsymbol{e} t \boldsymbol{a l}$. Leptospirose bovina na região sul do Estado do Rio Grande do Sul. A Hora Veterinária, ano 14, n.84, p.15-20, 1995.

CALDAS, E.M., TISHCENKO, L.M., PEREIRA FILHO, M. $\boldsymbol{e}$ al. Aglutininas antileptospira em hemo-soros de animais. Arq EMV-UFBa, v.2, n.1, p.83-98, 1977.

CALDAS, E.M., SAMPAIO, M.B., COSTA, J.A. Investigação comparativa de estirpes apatogênicas para o diagnóstico sorológica de leptospirose em animais Arq EMV-UFBa, v.10, n.1, p.14-47, 1986.

CAMPOS Jr., A.C.P., FRENEAU, G.E. Sorologia positiva para leptospira em rebanhos nos Municípios de Inhumas e Palmeiras-GO. In: CONGRESSO PANAMERICANO DE CIÊNCIAS VETERINÁRIAS, 15, Campo Grande, 1996. Anais... Campo grande : Sociedade Matogrossense do Sul de Medicina Veterinária. 1996. p.288.

CENTRO PANAMERICANO DE ZOONOSES. Procedimentos para estudios de prevalência de enfermidades crônicas en el ganado. Benos Aires : Ramos Mejia, 1973. 28p. (Nota técnica n. 18).

CISNEROS, M.A., RAMIREZ, R., TORRES, J. et al. Primer report en el México de L.interrogans serovariedad aislada de cerdos. In: CONGRESSO PANAMERICANO DE CIÊNCIAS VETERINÁRIAS, 15, Campo Grande, 1996. Anais... Campo Grande : Sociedade Matogrossense do Sul de Medicina Veterinária, 1996. p.280.

CORDEIRO, F., GUIDA, H.G., RAMOS, A.A. et al. Aglutininas antileptospira em soro de bovinos do Estado do Rio de Janeiro. Pesq Agrop Bras Série Vet, v.10, p.9-19, 1975.

DÓRIA, J.D., SANTANA, E.C. Leptospirose IV: Aglutininas antileptospira em soros bovinos no Estado da Bahia. Arq EMV-UFBa, v.1, n.1, p.74-79, 1976.

DÓRIA, J.D., VIEGAS, S.A.R.A., VIEGAS, E.A. et al. Investigação sorológica de doenças ligadas à esfera reprodutiva em bovinos, no Estado da Bahia, 1976. Arq EMV-UFBa, v.7, n.1, p.97-104, 1982.

ELLIS, W.A. Bovine leptospirosis in the tropics: prevalence, pathogenesis and control. Preventive Vet Med, v.2, p.411422, 1984

EZEH, A.O., ADDO, P.B., ADESIYUN, A.A et al.. Serological prevalence of bovine leptospirosrs in Plateau State, Nigéria. Rev Med Vet Pays Trop, v.42, n.4, p.505-508, 1990.

GUIMARÃES, M.C. Epidemiologia e controle da leptospirose em bovinos. Papel do portador e o seu controle terapêutico. Rev Fac Med Vet Zootec USP, v.19, n.2, p.202, 1982.

JARDIM, E.C., SILVA, R.L., ALMEIDA, M.M.R. et al. Aglutininas antileptospira em bovinos do Estado de Goiás, Anais da EMV-UFG, 1978. n.p. 
KURIBARA, I.Y, LANGONI, H., CABRAL, K.G. $\boldsymbol{e}$ t al Serological survey for leptospira and toxoplasma antibodies en capybaras (Hydrochoerus hydrochaeris). In: CONGRESSO PANAMERICANO DE CIÊNCIAS VETERINÁRIAS, 15, Campo Grande, 1996. Anais.. Campo Grande : Sociedade Matogrossense do Sul de Medicina Veterinária, 1996. p.287.

LANGENEGGER, J. Aborto causado por leptospiras-diagnóstico e medidas de controle da leptospirose em bovinos. Pesq Vet Bras, v.10, n.1/2, p.4-5, 1990.

LILENBAUM, W. Atualização em leptospiroses bovinas. Rev Bras Med Vet, v.18, n.1, p.9-13, 1996.

LOPEZ, J.I.M., POQUET, N.D. Diagnóstico serologico de leptospirosis bovina en la VIII region of Chile. AgroCiência, v.6, n.1, p.23-26, 1990.

LUNA, J.J.F., VARGAS, V.A.R., MOCTEZUMA, A.P. Detección de anticuerpos contra Leptospira interrogans en bovinos de hatos lecheros en el valle de Atlixco, Puebla, mediante la prueba de aglutinacion microscópica. Vet Méx, v.24, n.1, p.47-49, 1993.

MADRUGA, C.R., AYCARDI, E., PUTT, N. Freqüência de aglutininas anti-leptospira em bovinos de corte na região Sul de cerrado do Estado de Mato Grosso. Arq Esc Vet UFMG, v.32, n.2, p.245-249, 1980.

MANUAL DE CONTROLE DA LEPTOSPIROSE. Ministério da Saúde. Fundação Nacional de Saúde-Leptospirose. Centro Nacional de Epidemiologia. Coordenação de Controle de Zoonoses e Animais Peconhentos. 2 ed. rev. Brasília Fundação Nacional de Saúde, 1989. 98p.

MILLER, D.A., WILSON, M.A., BERAN, G.W. Survey to estimate prevalence of Leptospira interrogans infection in mature cattle in the United States. Am J Vet Res, v.52, n.11, p.1761-1768,1991.

MOREIRA, E.C., SILVA, J.A., VIANA, F.C. et al. Leptospirose Bovina: I- Aglutininas anti-leptospiras em soros sanguíneos de bovinos de Minas Gerais. Arq Esc Vet UFMG, v.31, n.3, p. $375-388,1979$.

OLIVEIRA, S.J. Presença de aglutininas antileptospira em suínos e bovinos, com e sem sinais de infecção, no Rio Grande do Sul. Boletim IPVDF, v.4, p.57-64, 1977.

ORDUÑA, G.R.S., SALAZAR, G.F., FLORES, T.M. $\boldsymbol{e}$ t al Freqüência de Leptospira interrogans em bovinos sacrificados en un hasto del Estado de México, México. In: CONGRESSO PANAMERICANO DE CIÊNCIAS VETERINÁRIAS, 15, Campo Grande, 1996. Anais... Campo grande : Sociedade Matogrossense do Sul de Medicina Veterinária, 1996. p.263.

PEREIRA, M.C., ROCHA, T. Infecção por Leptospira em bovinos soronegativos à brucelose em Portugal: um estudo adicional. Vet Port de Ciên Vet, v.86, n.498, p.90-98, 1991.

SANTA ROSA, C.A., CASTRO, A.F.P., TROISE, C. Isolamento de Leptospira icterohaemorragiae de bovino em São Paulo. Arquivo do IBSP, v.28, p.113-118, 1961.

STEELE, J.H., MILDRED, M.P.H., GALTON, M. et al. Leptospirosis as a world problem. Veterinary Medicine, v.3, n.11, p.517-527, 1957 .

VASCONCELLOS, S.A., BARBARINI JUNIOR, O.O., UMEHARA, O. et al. Leptospirose bovina, níveis de ocorrência e sorotipos predominantes dos Estados de Minas Gerais, São Paulo, Rio de Janeiro, Paraná, Rio Grande do Sul e Mato Grosso do Sul. Período de Janeiro a Abril de 1996. Arq Inst Biol, São Paulo, v.64, n.2, p.7-15, 1997.

VIEGAS, S.A.R.A. DÓRIA,J.D., ROCHA, J.V.N. et $\boldsymbol{a l}$. Aglutininas antileptospiras em soro de bovinos no Estado da Bahia. Arq EMV-UFBa, v.10, n.1, p.3-13, 1986.

Ciência Rural, v. 30, n. 5, 2000. 